\title{
A solute-binding protein for iron transport in Streptococcus iniae
}

\author{
Lili Zou, Jun Wang, Baofeng Huang, Mingquan Xie, Anxing Li
}

\begin{abstract}
Background: Streptococcus iniae (S. iniae) is a major pathogen that causes considerable morbidity and mortality in cultured fish worldwide. The pathogen's ability to adapt to the host affects the extent of infection, hence understanding the mechanisms by which S. iniae overcomes physiological stresses during infection will help to identify potential virulence determinants of streptococcal infection. Grow S. iniae under iron-restricted conditions is one approach for identifying host-specific protein expression. Iron plays an important role in many biological processes but it has low solubility under physiological condition. Many microorganisms have been shown to be able to circumvent this nutritional limitation by forming direct contacts with iron-containing proteins through ATPbinding cassette $(A B C)$ transporters. The $A B C$ transporter superfamilies constitute many different systems that are widespread among living organisms with different functions, such as ligands translocation, mRNA translation, and DNA repair.

Results: An ABC transporter system, named as mtsABC (metal transport system) was cloned from S. iniae HD-1, and was found to be involved in heme utilization. $m t s A B C$ is cotranscribed by three downstream genes, i.e., $m t s A$, $m t s B$, and $m t s C$. In this study, we cloned the first gene of the $m t s A B C$ transporter system $(m t s A)$, and purified the corresponding recombinant protein MtsA. The analysis indicated that MtsA is a putative lipoprotein which binds to heme that can serve as an iron source for the microorganism, and is expressed in vivo during Kunming mice infection by S. iniae HD-1.

Conclusions: This is believed to be the first report on the cloning the $A B C$ transporter lipoprotein from S. iniae genomic DNA. Together, our data suggested that MtsA is associated with heme, and is expressed in vivo during Kunming mice infection by S. iniae HD-1 which indicated that it can be a potential candidate for $S$. iniae subunit vaccine.
\end{abstract}

\section{Background}

Streptococcus iniae (S. iniae) is a hemolytic Gram-positive coccus that is a major pathogen of culture fish. It has been associated with disease outbreak in several species of freshwater and marine fish cultured worldwide, including tilapia [1,2], barramundi [3], channel catfish [4], hybrid striped bass [1,5], Japanese flounder [6,7], olive flounder [8], rabbitfish [9], and rainbow trout $[9,10]$. Streptococcal infection can lead to serious symptoms such as meningoencephalitis and generalized septicaemia with high mortality rates of up to $50 \%[9,11]$. S. iniae is also known to be an opportunistic pathogen

\footnotetext{
* Correspondence: lianxing@mail.sysu.edu.cn

Key Laboratory for Aquatic Products Safety of Ministry of Education/State

Key Laboratory of Bio-control, School of Life Sciences, Sun Yat-sen University, 135 Xingang West Road, Haizhu District, Guangzhou 510275, PR China
}

that can cause fulminant soft tissue infection in humans, such as bacteremic cellulitis, septicarthritis, and endocarditis [12]. Identifying potential virulence determinants of streptococcal infection will eventually help to the control and eradication of the disease.

Iron plays a significant role in many biological processes and is vital for several metabolic processes. Moreover, many proteins such as cytochromes and tricarboxylic acid metalloenzymes use iron as a cofactor [13]. Iron is also required for important cellular functions such as the transport and storage of oxygen and as a catalyst in electron transport processes [14]. The levels of several virulence determinants produced by bacterial pathogens, such as toxins and hemolysins, are depressed under iron-restricted conditions [15]. Despite its abundance in the natural environment, iron has low solubility under physiological
C Biomed Central 
conditions. Moreover, it may be associated with heme or hemo-proteins such as transferrin, lactoferrin, haptoglobin, hemoglobin, and ferritin and such forms do not readily support the growth of microorganisms. Many microorganisms circumvent this nutritional limitation by forming direct contacts with iron-containing proteins through ATP-binding cassette $(\mathrm{ABC})$ transporters.

The $A B C$ transporter superfamilies constitute many different systems that are widespread among living organisms and show different functions, such as ligands translocation, mRNA translation, and DNA repair. The general principle of $\mathrm{ABC}$ transport systems involves the ligands translocation through a pore formed by two integral membrane protein domains. This is accompanied by ATP hydrolysis through two nucleotide-binding domains associated with the cytoplasmic side of the pore. In bacteria, ligand translocation is preceded by interaction with an accessory component, i.e., the periplasmic-binding protein [16].

In this study, an $A B C$ transporter member, named as $m t s A B C$ (metal $A B C$ transport system) was cloned from $S$. iniae HD-1 which is cotranscribed by three genes and was shown to share amino acid sequence homology with the metal ABC transport proteins of other Grampositive and Gram-negative bacteria. BLAST-mediated sequences similarity searches of the derived amino acid sequences of the $m t s A B C$ operon indicated that $m t s A$ encodes a metal solute-binding lipoprotein, $m t s B$ encodes an ATP-binding protein (ATPase), and $m t s C$ encodes a transmembrane permease protein. Our data showed that MtsA is a lipoprotein, and associated with heme. Moreover, this protein is expressed in vivo during Kunming mice infection by $S$. iniae HD-1. These results provide information on the role of MtsA in heme utilization and the possibility of using MtsA as an effective $S$. iniae vaccine candidate.

\section{Results}

Cloning and reverse transcriptase-PCR analysis of $m t s A B C$ To clone $m t s A B C$ from $S$. iniae HD-1, primers designed based on the conserved regions of the published amino acid sequence of metal $\mathrm{ABC}$ transporter were used. The PCR products from genomic DNA template were subsequently sequenced by Invitrogen Corporation. The results showed that the ORFs of $m t s A$ [GeneBank: HQ170628], $m$ ts $B$ [GeneBank: HQ170629], $m t s C$ [GeneBank: HQ170630], $m t s A B$, and $m t s B C$ had 930, 729, 852,1724 , and 1574 bp respectively. Reverse transcriptase-PCR analysis confirmed that the $m t s A$ gene is the first of three contiguous ORFs that are preceded by a potential promoter region. These three genes are transcribed in the same direction and have very short intergenic sequences, with transcription terminating between $m t s A$ and $m t s B$, suggesting that these genes constitute a single transcriptional unit (Figure 1). No corresponding PCR products were obtained with the same mRNA sample as the template, indicating that the RNA sample was not contaminated with DNA.

\section{Sequence analysis of $m t s A B C$}

$A B C$ systems are widespread among living organisms and have been detected in all genera of the three kingdoms of life. These systems show remarkable conservation in the primary sequence of the cassette and in the organization of constitutive domains or subunits $[17,18]$. All ABC systems share a highly conserved ATP-hydrolyzing domain (nucleotide-binding domain [NBD]) that is unequivocally characterized by three short sequence motifs, i.e., Walker A, Walker B, and a signature motif that is unique to $\mathrm{ABC}$ proteins and is located upstream of the Walker B motif [19-24]. BLAST of the derived amino acid sequences of the $m t s A B C$ operon indicated that $m t s A$ encodes a metal solute-binding lipoprotein (MtsA, 309 residues), $m t s B$ encodes an ATP-binding protein (MtsB, 242 residues), and $m t s C$ encodes a transmembrane permease protein ( $\mathrm{MtsC}, 283$ residues). The closest homologs for these proteins are putative metal ABC transporter proteins encoded by the $m t u$ locus of Streptococcus uberis 0140J and the mts locus of Streptococcus equi subsp. zooepidemicus MGCS10565 (Additional file 1, Table S1, and Figure 2). $m$ ts $A$ contains a helical backbone metal receptor (TroA-like domain) that functions in the $\mathrm{ABC}$ transport of ferric siderophores and metal ions such as $\mathrm{Fe}^{3+}, \mathrm{Mn}^{2+}, \mathrm{Cu}^{2+}$, and/or $\mathrm{Zn}^{2+}$ (Additional file 1, Table S2). $m t s B$ contains Walker site A, Walker site B, a signature sequence, and the $4^{\text {th }}$ motif as defined by Linton \& Higgins [25]. $m t s C$ contains eight transmembrane subunits (TMs) of the periplasmic-binding protein (PBP)-dependent $\mathrm{ABC}$ transporters that are possibly involved in the uptake of siderophores, heme, vitamin $\mathrm{B}_{12}$, or divalent cations (Additional file 1, Table S2). Based on these observations, we concluded that $m t s A B C$ is a member of the ABC transporter systems.

Three patterns of signal peptide (Additional file 1, Table S3) were used to identify bacterial lipoproteins from bioinformatics data [26]. To characterize the MtsA protein, the ScanProsite analysis was performed. The results indicated that the sequence of MtsA showed similarity to the following 2 patterns: residues D1 to D24 (MFKKISLAFAMLLSIFCITACSSQ) hit the G+LPPv2 pattern, and residues D17 to D21 (CITAC) hit the PS51257 pattern (Figure 3A) [27], which suggested that MtsA is a lipoprotein. $m$ ts $A$ contains an lipoprotein peptidase cleavage site signal sequence as defined by Linton \& Higgins [25]. To confirm that MtsA is a lipoprotein, the crude cell lysate of $S$. iniae HD-1 was mixed with Triton X-114, and the detergent phase was analyzed by 


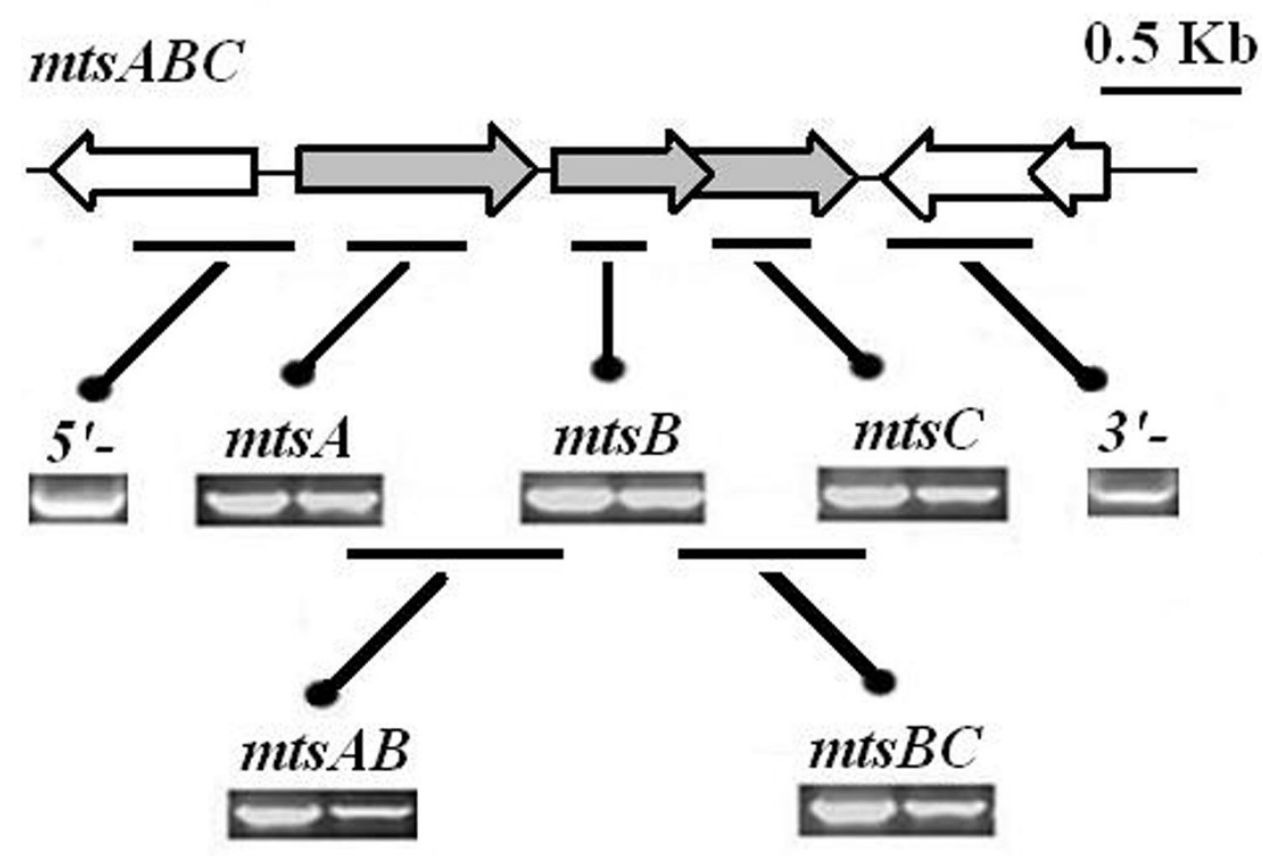

Figure 1 Reverse transcriptase-PCR analysis demonstrates a polycistronic transcript of $m$ ts $A B C$ mRNA. Total RNA from $S$. iniae HD- 1 was reverse transcribed into CDNA, and PCR was performed with ORF-specific primers. Each box contains products with the same primer pairs. For $P C R$, S. iniae HD-1 genomic DNA was used as the template (on the left), and for reverse transcriptase-PCR, S. iniae HD-1 RNA was used as the template (on the right).

western blotting using rabbit anti-MtsA antibodies (Figure 3B). The results showed that MtsA protein was extracted by Triton X-114. Together, the results indicated that MtsA protein is a lipoprotein.

\section{Purification of recombinant MtsA}

To be able to further characterize MtsA, we first expressed recombinant MtsA consisting of amino acid residues D27 to D310 that lacked the putative signal sequence. Briefly, $m t s A$ gene was cloned and the PCR product was isolated from the plasmid after a double digestion with restriction enzymes BamHI and XhoI, and ligated into the compatible site of pET-32a-c (+) Vector to yield recombinant protein MtsA. The expressed MtsA had a molecular mass of $49.5-\mathrm{kDa}$ (Figure 4) with a tag from Trx.Tag to EcoR $V$ of pET$32 \mathrm{a}-\mathrm{c}(+)$, which has a molecular weight of 17.7-kDa. The expression level of MtsA peaked after induction with $1 \mathrm{mM}$ IPTG at $37^{\circ} \mathrm{C}$ for $4 \mathrm{~h}$. The MtsA protein was purified from E. coli BL21 (DE3) under native

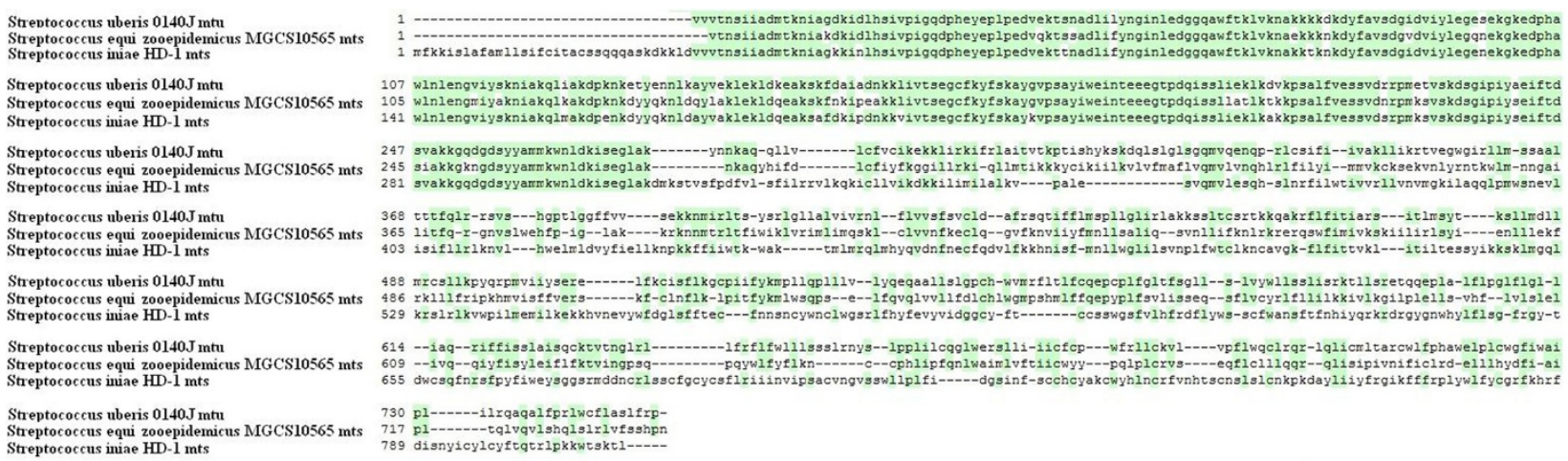

Figure 2 Sequence alignment of MtsABC and its homologues. The amino acid sequences were aligned using the the SECentral Align Multi 4 program. Dark shading represented identical amino acid residues. 


\section{A MtsA MFKKISLAMLLSIfCrtacsSQ(residues 1-24) \\ $<$ [MV]-X(0,13)-[RK]-\{DERK\}(6,20)-[LVMFESTAGPC]-[LVLAMFTG]-[MMSTAGCP]-[AGS]-C (G+LPPV2 pattern)}

CrTAC(residues 17-21)

[DERK)(6)-[LIVMFWSTAG](2)-[LIVMFYSTAGCQ]-[AGS]-C

(PS51257 pattern)

No hit

$<$ [MV]-X(0,13)-[RK]-\{DERKQ\}(6,20)-[LIVMFESTAG]-[LVAM]-[IVMSTAFG]-[AG]-C

(G+LPP pattern)

B

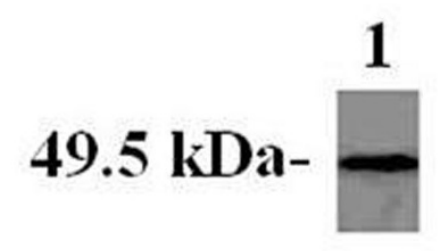

Figure 3 Analysis of the lipoprotein sequence patterns of MtsA by ScanProsite and the western blotting. (A) The mtsABC lipoprotein waS assessed by ScanProsite. The results showed that amino acid residues D1 to D24 (MFKKISLAFAMLLSIFCITACSSQ) hit G+LPPV2 pattern, and amino acid residues D17 to D21 (CITAC) hit PS51257 pattern. The symbol " $<$ " indicates that the pattern is restricted to the N terminus, and X is any amino acid. (B) Western blotting analysis results of the lipoproteins extracted with Triton X-114.

condition $\mathrm{n}$ the soluble form and immunized the New Zealand white rabbits. The results showed that the rabbit anti-MtsA antibody titers increased from essentially zero to 1:50,000 after four rounds of immunization (Additional file 1, Table S4). The western blotting analysis was performed to show the specificity of immunized sera against purified MtsA (Figure 4, and Additional file 2, Figure S3-4).

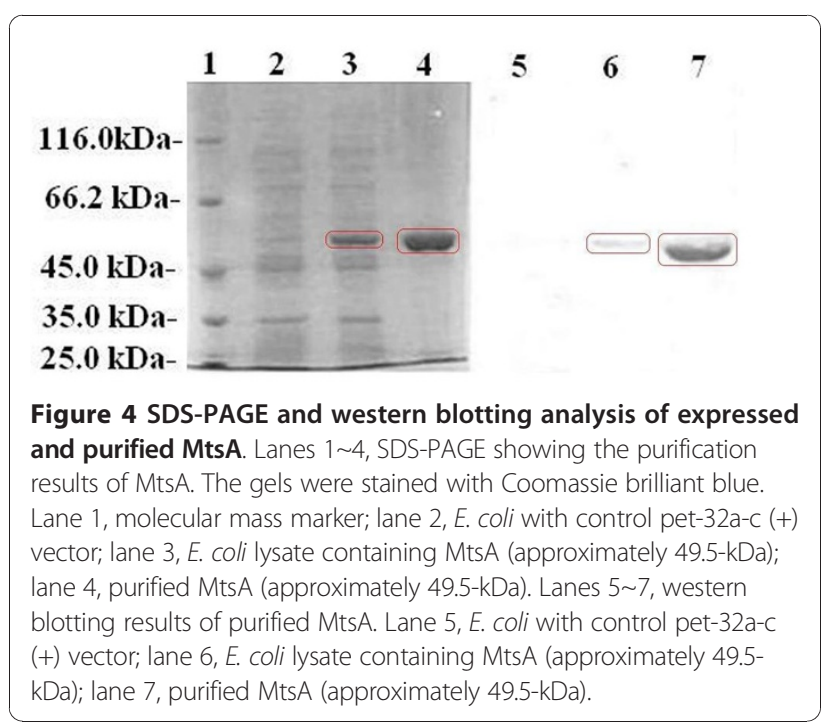

Subcellular localization of MtsA

To determine the subcellular localization of MtsA, the western blotting was performed with the cellular fractions of S. iniae HD-1 using rabbit anti-MtsA antibodies (Figure 5A). MtsA was detected in the particulate fraction of the cells when the cellular fractions were prepared by centrifugation of the crude cell lysate (the first treatment). MtsA was found to be associated with the protoplast and cell wall extracts when the cellular fractions were prepared by protoplast formation. After separation of the protoplasts, MtsA was detected in the particulate fraction (the second treatment).

To detect surface exposure of MtsA, cells of S. iniae HD-1 cells were harvested, washed, centrifuged, and resuspended in PBS. The cells were subjected to proteinase $\mathrm{K}\left(5 \mu \mathrm{g} \mathrm{ml}^{-1}\right)$ treatment with gentle agitation at room temperature for $1 \mathrm{~h}$, and the cells were collected. Western blotting showed that peptide fragments in the cells can be detected after $1 \mathrm{~h}$ incubation with proteinase $\mathrm{K}$. However, when the cell wall extracts and protoplasts were used in the experiment, it were completely hydrolyzed and no peptide fragments were detected (Figure 5B). Together, this result indicated that MtsA is not exposure on surface, but is on the outside of the cytoplasmic membrane and is buried inside the cell wall. 


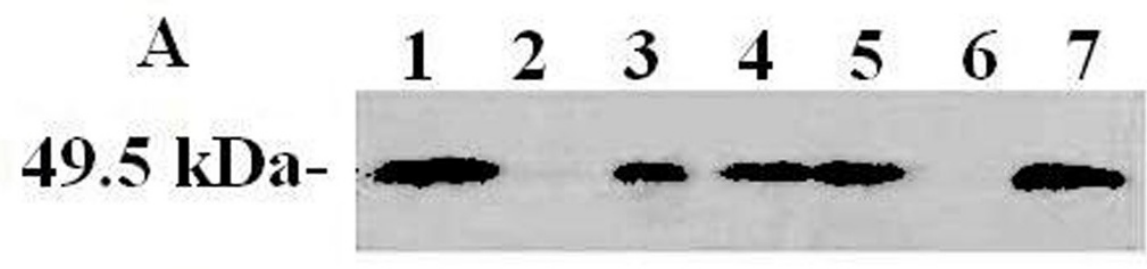

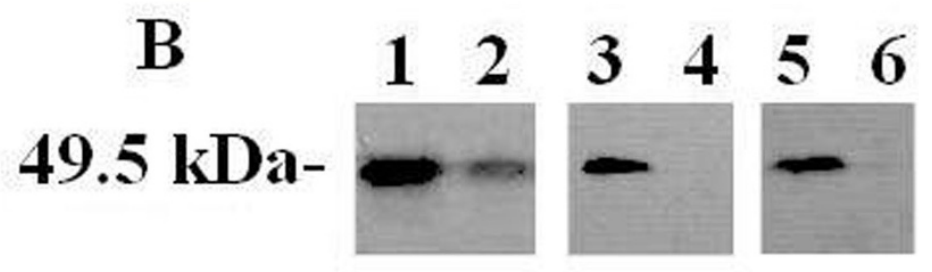

Figure 5 Detection of the subcellular localization of MtsA in S. iniae HD-1 by western blotting. (A) The cellular fractions of S. iniae HD-1 and rabbit anti-MtsA antibodies were used for the western-blot assay. Lane 1, S. iniae HD-1 lysate; lane 2, soluble fraction of cells; lane 3, particulate fraction of cells; lane 4, cell wall extracts; lane 5, protoplast; lane 6, particulate fraction of protoplasts; and lane 7, soluble fraction of protoplasts. (B) Surface exposure of MtsA. Cells (lanes 1 and 2), cell wall extracts (lanes 3 and 4), and protoplasts (lanes 5 and 6) of S. iniae HD-1 were treated with proteinase $\mathrm{K}$ and analyzed by western blotting. Lanes 1, 3 and 5 show the untreated control, while lanes 2, 4 and 6 show samples treated with proteinase $\mathrm{K}$ for $1 \mathrm{~h}$.

\section{MtsA had heme-binding activity}

To examine whether heme is the chromophore associated with MtsA, the pyridine hemochrome assay was performed [28]. The UV-visible absorption spectrum of purified MtsA exhibited peaks at 275, 420, 525, and $560 \mathrm{~nm}$, which were identical to those obtained from purified KatG, a well-known heme-containing protein with spectral peaks at 418, 524, and $556 \mathrm{~nm}$. The molar ratio of associated heme to purified MtsA was 0.806 (Figure 6), this value is consistent with the hypothesis that one protein molecule is associated with one heme molecule.

To determine whether iron is present and its amount in purified MtsA, the ICP-AES analysis were performed. The results showed that Fe was present (Additional file 1, Table S5) in purified MtsA; however, four other bivalent metallic elements $\mathrm{Ca}, \mathrm{Mg}, \mathrm{Zn}$ and $\mathrm{Mn}$ were not detected. The amount of iron present in purified MtsA $(20 \mu \mathrm{M})$ was $1.43,1.38$, and $1.33 \mathrm{mg} \mathrm{L}^{-1}$, in three independent purification experiments respectively.

\section{In vivo production of MtsA during S. iniae HD-1 infection}

To determine whether MtsA is produced in vivo during $S$. iniae infection, we infected Kunming mice with S. iniae HD-1 and performed western blotting analysis with purified MtsA to determine the presence of antiMtsA antibodies in infected sera (Figure 7). The results indicated that MtsA is produced in vivo during experimental S. iniae HD-1 infection.

\section{Discussion}

Heme is an important nutrient for several bacteria and can serves as a source of essential iron. The most abundant source of iron in the body is heme, so it is not surprising to find that pathogenic bacteria can use heme as an iron source [29]. The presence of the central iron atom in heme allows it to undergo reversible oxidative change and act as a virulence-regulated determinant [30-36]. It is necessary for bacterial pathogens to acquire sufficient iron from their surroundings, and scavenging heme from the environment requires much less effort than synthesizing it de novo [30,34].

Acquiring iron from the micro-environment is important for the growth of bacterial pathogens. Pathogens often use low environmental iron levels as a signal to induce virulence genes [14]. Many pathogenic bacteria secrete exotoxins, proteases, and siderophores to rapidly increase the local concentration of free heme [37], and it is common for pathogens to directly acquire iron from host iron-binding proteins by using receptor-mediated transport systems specific for host-iron complexes [38].

To define the role of MtsA in heme utilization, the binding activity and subcellular localization of purified MtsA were investigated. The coding sequence of $m t s A$ was cloned into the expression vector pet-32a-c (+). The major induced protein in E. coli (BL21) migrated as a 49.5-kDa band in SDS-PAGE gels; this size is consistent with the predicted molecular mass of MtsA. The 49.5$\mathrm{kDa}$ MtsA was purified by $\mathrm{Ni}^{2+}$ affinity chromatography 

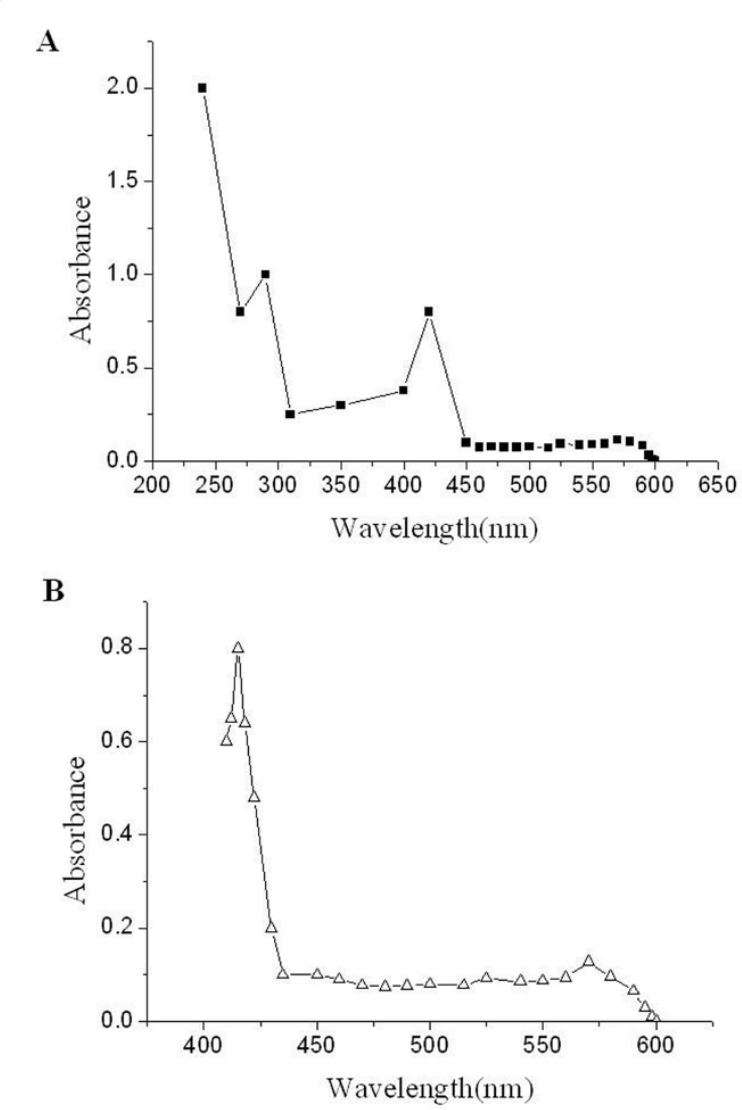

Figure 6 Detection of the heme-binding activity of purified MtsA by the pyridine hemochrome assay. (A) UV-visible absorption spectrum of $20 \mu \mathrm{M}$ purified MtsA ( line) in $50 \mathrm{mM}$ Tris$\mathrm{HCl}(\mathrm{pH}$ 8.0). (B) UV-visible absorption spectrum of $20 \mu \mathrm{M}$ purified KatG ( $\triangle$ line) in $50 \mathrm{mM}$ Tris-HCl $(\mathrm{pH} 8.0)$.

and reacted with anti-MtsA antibodies from infected mice to confirm the in vivo production of MtsA. The UV-visible absorbance spectrum of KatG is a typical heme-containing protein, and the results of the pyridine hemochrome assay indicated that MtsA is associated with heme. Moreover, measurements of the iron level by ICP-AES indicated that purified MtsA is a holoprotein that is associated with iron.

In general, there are four major types of cell surface display proteins in Gram-positive bacteria, which are as follows: proteins anchored to the cytoplasmic membrane by hydrophobic transmembrane domains; lipoproteins that are covalently attached to membrane lipids after cleavage by signal peptides II; proteins that contain the C-terminal LPXTG-like motif and are covalently attached to peptidoglycan by sortase; and proteins that recognize some cell wall components by specific domains [39]. ABC transporters are integral membrane proteins that transport diverse substrates across lipid bilayers [40]. In bacteria, ABC

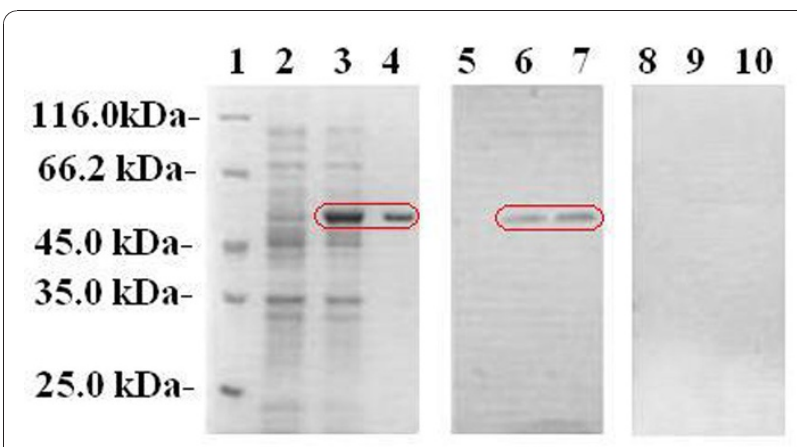

Figure 7 Western blotting analysis of anti-MtsA antibodies in infected sera from Kunming mice with S. iniae HD-1 infection. SDS-PAGE analysis showing the purification results of MtsA. The gel was transferred to a nitrocellulose membrane and blotted with infected sera from mice. The gels were stained with Coomassie brilliant blue. Lane 1, molecular mass marker; lane 2, E. coli with control pet32a-c (+) vector; lane 3, E. coli lysate containing MtsA (approximately 49.5-kDa); lane 4, purified MtsA (approximately 49.5-kDa); lanes 5 7, western blot results of infected sera, lanes $8 \sim 10$, western blot results of control sera; lanes 5 and 8, western blot results of $E$. coli with the control vector; lanes 6 and 9, E. coli lysate containing MtsA, and lanes 7 and 10, purified MtsA (approximately 49.5-kDa).

transporters catalyze the uptake of essential nutrients or the extrusion of toxic substances [41]. ABC importers, present only in prokaryotes, require a binding protein that delivers the captured substrate to the external face of the transporter [42]. As MtsA is a solute-binding protein of the $\mathrm{ABC}$ transporter, its major function is presumed to be the capture and transfer of iron compounds to the downstream gene of the iron transport system of S. iniae HD-1. The signal peptide pattern analysis and Triton X-114 extraction results confirmed that MtsA is a lipoprotein. This result is reliable because the original G+LPP pattern was present in the analysis of the signal peptide features of 33 experimentally verified lipoproteins. Lipoproteins in Gram-positive bacteria are cell envelope proteins anchored to the outer leaflet of the plasma membrane. Lipid modification is achieved through covalent addition of a diacylglyceride to an indispensable cysteine residue in the lipoprotein signal peptide that provides a common anchoring mechanism for what is now recognized as an abundant and functionally diverse class of peripheral membrane proteins [40]. In Gram-positive bacteria, substrate-binding proteins of $\mathrm{ABC}$ transporters are typically lipoproteins $[41,42]$, and the western blotting results is consistent with the notion that MtsA is an $\mathrm{ABC}$ transporter lipoprotein $[43,44]$.

The results of this study indicated that $m t s A B C$ is a member of the $A B C$ transporter family. MtsA protein is a solute-binding protein that can bind to heme and facilitate the latter's use as a substrate by the $S$. iniae. Western blotting indicated that MtsA is produced 
in vivo during experimental $S$. iniae HD-1 infection, and MtsA may be a potentially useful $S$. iniae protein vaccine candidate. Further studies are needed to clarify the role of MtsA in the utilization of other iron compounds and other important cations, and to establish its candidacy as a useful $S$. iniae vaccine component.

\section{Conclusions}

In summary, this study presents MtsA as a novel solutebinding protein that can contribute to iron transport. This is the first $\mathrm{ABC}$ transporter member to be identified from S. iniae. We have shown that MtsA is a lipoprotein which can bind to heme, and is expressed in vivo during Kunming mice infection by $S$. iniae HD-1. More importantly, this is the first report on the cloning of $\mathrm{ABC}$ transporter lipoprotein from $S$. iniae genomic DNA, and its immunogenicity is indicative of its possible use as an $S$. iniae subunit vaccine.

\section{Methods}

\section{Bacterial strains and growth conditions}

Streptococcus iniae HD-1 was isolated from Threeband sweetlips (Plectorhynchus cinctus) from Guangdong province, PRC. The microorganism was stored in our lab and cultured according to the methods described by Zhou et al [45]. Briefly, S. iniae isolate HD-1 cells were grown in brain heart infusion broth (BHI, Oxoid Ltd.), and $\mathrm{BHI}$ broth with $1.5 \%$ agar (Guangdong Huankai Microbial Sci. \& Tech, Co., Ltd.) was used as the solid medium. Escherichia coli DH5 $\alpha$ and BL21 (DE3) strains (Beijing Newprobe Biotechnology Co., Ltd.) were used for gene cloning and protein expression, respectively.

\section{Cloning and reverse transcription analysis of $m t s A B C$} Genomic DNA was extracted from the S. iniae HD-1 strain using the Wizard genomic DNA purification kit (Promega Co., Ltd.), as recommended by the manufacturer, and the material was quantified by measuring the absorbance at $260 \mathrm{~nm}$. PCR was carried out with $1 \mu \mathrm{g}$ of DNA using the primers listed in Additional file 1, Table S6. The primers were designed based on the conserved regions of the published amino acid sequence of metal ABC transporter (Additional file 1, Table S6-1), and the full-length product was obtained by SiteFindingPCR (Additional file 1, Table S6-2, 6-3), as described by Tai et al [46]. The PCR products were sequenced to rule out spurious mutations (Invitrogen Co., Ltd.).

S. iniae HD-1 cells grow to the logarithmic phase were harvested by centrifugation, and total RNA was extracted by the Pure Yield ${ }^{\text {ma }}$ RNA midiprep system (Promega, USA, Co., Ltd.). Total RNA was then incubated with RNase $\mathrm{I}$ at $37^{\circ} \mathrm{C}$ for $30 \mathrm{~min}$ to remove the contaminating genomic DNA. The material was quantified spectrophotometrically by ultraviolet absorption spectrometry (CE2302, Gene Quest), and its integrity was verified on a $0.8 \%$ agarose gel. First-strand cDNA was synthesized from $1 \mu \mathrm{g}$ total RNA using the first-strand cDNA synthesis kit with ReverTra Ace- $\alpha$ reverse transcriptase (Toyobo Co., Ltd.). The cDNA synthesized above was used as the template to amplify genes using the ORF-specific primers listed in Additional file 1, Table S7, and the PCR products were sequenced at Invitrogen Corporation to confirm their specificity.

\section{Expression of recombinant MtsA}

The genomic DNA of S. iniae HD-1 was used as the template to amplify the gene encoding amino acids 27 to 310 of $m t s A$ together with primers 5'-GCGGG ATCCGCCTCTAAAGATAAG-3' (underlined nucleotides refer to the $B a m H I$ restriction site-initiation codon) and 5'-GCGCTCGAGTTATTTTGCTAAG CCTTCTGAA-3' (underlined nucleotides refer to the XhoI restriction site-stop codon), which were designed from the sequenced $m t s A$ gene. The protein from this cloned amino acid sequence lacks the presumed signal sequence (amino acids 1 to 26). The cloned amino acid fragment was sequenced by Invitrogen Corporation to rule out the possibility of spurious mutations. Recombinant MtsA was purified from E. coli BL21 (DE3) under native conditions using nickel-nitrilotriacetic acid (NiNTA) columns (Qiangen, USA) as recommended by the manufacturer. The protein purified by this protocol was free of contaminating proteins, as assessed by sodium dodecyl sulfate-polyacrylamide gel electrophoresis (SDSPAGE). It was quantified by the Bradford assay (CE2302, Gene Quest) using BSA $\left(0.5 \mathrm{mg} \mathrm{ml}^{-1}\right)$ as the standard. Specific fractions were then pooled.

\section{Preparation of anti-MtsA antibodies}

Anti-sera against histidine-tagged MtsA were prepared in male New Zealand white rabbits $(2.2 \mathrm{~kg})$, and approval from the Animal Ethics Committee of Life Sciences Institute was obtained prior to using the animals for research. The experiments were performed as stipulated by the China State Science and Technology Commission [47]. Rabbits were purchased from Guangdong Laboratory Animals Research Center and acclimatized for 2 weeks in the laboratory of the Life Science Institute prior to use. The rabbits were maintained at the SPF animal center and fed twice daily. They were immunized with $850 \mu \mathrm{g}$ purified MtsA in $100 \mu \mathrm{l}$ complete Freund adjuvant (Sigma-Aldrich, Inc.) and then boosted with $170 \mu \mathrm{g}$ MtsA in $100 \mu \mathrm{l}$ incomplete Freund adjuvant (SigmaAldrich, Inc.) three times at an interval of 15 days. The sera were collected 1 day before the first immunization 
and 7 days after each booster dose. Purified MtsA and collected sera were used to determine the rabbit antiMtsA antibody titer by the dot blotting assay.

\section{Extraction of the $S$. iniae HD-1 lipoprotein}

TritonX-114 was used to extract the S. iniae HD-1 lipoprotein, according to the method modified by Cockayne et al $[48,49]$. Briefly, S. iniae HD-1 cells were cultured, harvested, suspended, and sonicated. Next, $100 \mu \mathrm{l}$ of $10 \%$ TritonX-114 in PBS was added to $2 \mathrm{ml}$ of HD-1 cells lysate and incubated at $4^{\circ} \mathrm{C}$ for $2 \mathrm{~h}$. After centrifugation at $13,000 \times g$ for $10 \mathrm{~min}$, the supernatant was transferred to a fresh tube and incubated at $37^{\circ} \mathrm{C}$ for $30 \mathrm{~min}$ to allow phase separation. The detergent layer was retained after centrifugation at $13,000 \times g$ for $10 \mathrm{~min}$ at room temperature, washed with $1 \mathrm{ml}$ PBS at $4^{\circ} \mathrm{C}$ for $1 \mathrm{~h}$, and separated from the aqueous phase after incubation at $37^{\circ} \mathrm{C}$ [50]. The detergent layer was diluted 1:1 with water, and analyzed by western blotting using the rabbits anti-MtsA antibodies.

\section{Preparation of MtsA cellular fractions}

To determine the subcellular localization of MtsA in S. iniae HD-1, the cellular fractions were subjected to two different treatments. In the first treatment procedure, S. iniae HD-1 cells were cultured overnight in $50 \mathrm{ml} \mathrm{BHI}$, harvested, and resuspended in one-tenth volume of Tris buffer (1 M, pH 7.4), and disrupted by sonication ( $300 \mathrm{~W}, 5 \mathrm{~min}$ ). After removing unbroken cells by centrifugation at $10,000 \times g$, the crude cell lysate was further centrifuged at $248,000 \times g$ for $1 \mathrm{~h}\left(\mathrm{Optima}^{\mathrm{Tm}} \mathrm{L}\right.$ 100XP ultracentrifuge, Beckman Coulter). The supernatant and pellet were used as the soluble and particulate fractions of S. iniae cells, respectively [51]. In the second treatment procedure, the cellular fractions were obtained from S. iniae HD-1 by centrifugation using the protocol of Homonylo-McGavin \& Lee [52,53]. Briefly, S. iniae HD-1 cells were grown overnight in $30 \mathrm{ml} \mathrm{BHI}$ and then washed by centrifugation at $4^{\circ} \mathrm{C}$ in a buffer composed of ice-cold $20 \mathrm{mM}$ Tris and $1 \mathrm{mM} \mathrm{MgCl}$ (pH 7.0). The cell pellets were resuspended and incubated for $90 \mathrm{~min}$ in $0.3 \mathrm{ml}$ of protoplast buffer (150 $\mu \mathrm{l} 60 \%$ raffinose (Beijing Newprobe Biotechnology Co., Ltd.), $15 \mu \mathrm{l} 1 \mathrm{M}$ Tris (pH 7.4), $6 \mu \mathrm{l}$ $100 \mathrm{mM}$ phenyl-methyl sulfonyl fluoride (MBchem, Inc.), $3 \mu \mathrm{l} 1 \mathrm{M} \mathrm{MgCl}_{2}, 15 \mu \mathrm{l} 25,000 \mathrm{U} \mathrm{ml}^{-1}$ mutanolysin (SigmaAldrich, Inc.), $15 \mu \mathrm{l} 270,000 \mathrm{U} \mathrm{ml}^{-1}$ lysozyme, and $96 \mu \mathrm{l}$ $\mathrm{dd}_{2} \mathrm{O}$ ). The cell wall extracts were separated from the spheroplasts by centrifugation at $10,000 \times g$ for $10 \mathrm{~min}$. The pelleted protoplasts were washed, suspended in $2 \mathrm{ml}$ PBS-sucrose buffer, and disrupted by sonication, as described above. The supernatant and pellet obtained after centrifugation at $248,000 \times g$ for $1 \mathrm{~h}$ were used as the soluble and particulate fractions of the protoplasts, respectively. All cellular fractions were analyzed by western blotting using the rabbit anti-MtsA antibodies.

\section{Detection of the heme-binding activity of MtsA}

The pyridine hemochrome assay [28] was used to analyze heme binding to MtsA. Purified MtsA in $750 \mu \mathrm{l}$ of $10 \mathrm{mM}$ Tris- $\mathrm{HCl}(\mathrm{pH} 8.0)$ was mixed with $170 \mu \mathrm{l}$ of pyridine (Sigma-Aldrich, Inc.), $75 \mu \mathrm{l}$ of $1 \mathrm{~N} \mathrm{NaOH}$, and $2 \mathrm{mg}$ of sodium hydrosulfite (Beijing Newprobe Biotechnology Co., Ltd.), and heme content was determined by measuring the absorbance (-, black square) at $418 \mathrm{~nm}$ with a UV-visible spectrophotometer (Uvmini-1240, Shimadzu). Purified catalase-peroxidase (KatG, Beijing Newprobe Biotechnology Co., Ltd.), a known heme-containing protein, was used as the positive control ( $\Delta$, white triangle) [54].

\section{Measurement of iron in MtsA by ICP-AES}

The levels of $\mathrm{Fe}, \mathrm{Zn}, \mathrm{Ca}, \mathrm{Mg}$, and $\mathrm{Mn}$ in purified MtsA were determined by inductively coupled plasma-atomic emission spectrometry (ICP-AES) using an IRIS (HR) ICPAES instrument [55]. Briefly, $0.1 \mathrm{~g}$ purified MtsA was immersed in $15 \mathrm{ml}$ nitric acid in an electric cooker. After $3 \mathrm{~h}$ nitrification, $1 \mathrm{ml}$ perchloric acid was added and treated for $1 \mathrm{~h}$. The liquid was filter sterilized and analyzed by ICP-AES. A sample lacking purified MtsA was used as the negative control. To achieve contamination-deprivation conditions, all utensils were soaked overnight in nitric acid, and rinsed 6 times with $d_{d d} \mathrm{H}_{2} \mathrm{O}$.

\section{Detection of anti-MtsA antibodies in sera from Kunming} mice that were experimentally infected with $S$. iniae HD-1 To detect the presence of specific anti-MtsA antibodies in the sera from Kunming mice, 10 male Kunming mice (20 $\pm 2 \mathrm{~g}$ ) were purchased from Guangdong Laboratory Animals Research Center, and approval from the Animal Ethics Committee of Life Sciences Institute was obtained prior to using the animals for research. The experiments were performed as stipulated by the China State Science and Technology Commission [47]. Mice were acclimatized at the SPF animal center and fed twice daily for 2 weeks in the laboratory of the Life Science Institute prior to use. Each mouse was injected with $100 \mu \mathrm{l}$ of 6.2 $\times 10^{8} \mathrm{CFU} \mathrm{m}{ }^{-1} S$. iniae HD-1 cells, and the infected sera were collected 10 days post infection. The infected sera and purified MtsA were used in dot-blot and westernblot assays. The sera from 10 Kunming mice injected with PBS were used as the negative control.

\section{Statistical analysis}

The nucleotide and deduced amino acid homology analysis of $m t s A B C$ was carried out by ClustalX 1.83 and NCBI BLAST http://blast.ncbi.nlm.nih.gov/Blast.cgi. The presumed signal sequence was predicted by the signalP 
3.0 Server http://www.cbs.dtu.dk/services/SignalP/. The theoretical $\mathrm{pI} / \mathrm{MW}$ was analyzed by the ExPASy Compute $\mathrm{pI} / \mathrm{MW}$ tool http://www.expasy.org/tools/pi_tool. html. The main domains of $m t s A B C$ were detected by the SMART software http://smart.embl-heidelberg.de/. The amino acid sequences were aligned using the SECentral Align Multi 4 program. To determine whether $m t s A B C$ is a Lipoprotein, its sequence was assessed by the ScanProsite analysis software http://www.expasy.ch/ tools/scanprosite/. All statistical analyses were performed using the SPSS 16.0 software (SPSS Inc., USA).

\section{Additional material}

Additional file 1: Tables 1-7. Microsoft word file containing Tables 1-7 as individual tab-accessible tables within a single file (Supplemental Tables 1-7)

Additional file 2: Figures 1-4. Microsoft word file containing Figures 1 2, 3, 4 as individual tab-accessible figures within a single file (Supplemental Figures 1-4).

\section{Acknowledgements}

Project support was provided in parts by grants from Key Projects in the National Science \& Technology Pillar Program in the Eleventh Five-year Plan Period (2007BAD29B05) to Dr. An-Xing Li. Project support was provided in parts by grants from Chongqing Engineering Technology Research Centre of Veterinary Drug (CSTC, 2009CB1010) to Dr. Lili Zou.

We thank Prof. Shaoping Weng and Drs. Lichao Huang, Xiangyun Wu, Yangsheng Wu, Jianfeng Yuan, and Suming Zhou for their helpful technical advice. We also thank Dr. Shenquan Liao for providing plasmid pet-32a-c (+) used in this study, and the professional copyediting service from the International Science Editing

\section{Authors' contributions}

LLZ carried out the molecular genetic studies, participated in the sequence alignment studies, performed the statistical analysis, and drafted the manuscript. JW carried out the function studies and participated in the sequence alignment studies. HBF carried out the infection assay. MQX conceived of the study and participated in its design and coordination. AXL participated in the conceived of the study and helped to draft the manuscript. All authors read and approved the final manuscript.

Received: 13 August 2010 Accepted: 1 December 2010 Published: 1 December 2010

\section{References}

1. Shoemaker CA, Klesius PH, Evans JJ: Prevalence of Streptococcus iniae in tilapia, hybrid striped bass, and channel catfish on commercial fish farms in the United States. Am J Vet Res 2001, 62:174-177.

2. Perera RP, Johnson SK, Collins MD, Lewis DH: Streptococcus iniae associated with mortality of Tilapia nilotica $\times T$. aurea hybrids. J Aquat Anim Health 1994, 6:335-340.

3. Bromage ES, Owens L: Infection of barramundi Lates calcarifer with Streptococcus iniae: effects of different routes of exposure. Dis Aquat Org 2002, 52(3):199-205.

4. Stoffregen DA: Initial disease report of Streptococcus iniae infection in hybrid striped (sunshine) bass and successful therapeutic intervention with the fluoroquinolone antibacterial enrofloxacin. J World Aquac Soc 1996, 27(4):420-434.

5. Nguyen HT, Kanai K: Selective agars for the isolation of Streptococcus iniae from Japanese flounder. Paralichthys olivaceus, and its cultural environment. J Appl Microbiol 1999, 86(5):769-776.
6. Nguyen HT, Kanai K, Yoshikoshi K: Ecological investigation of Streptococcus iniae in cultured Japanese flounder (Paralichthys olivaceus) using selective isolation procedures. Aquaculture 2002, 205:7-17.

7. Nho SW, Shin GW, Park SB, Jang HB, Cha IS, Ha MA, Kim YR, Park YK, Dalvi RS, Kang BJ, Joh SJ, Jung TS: Phenotypic characteristics of Streptococcus iniae and Streptococcus parauberis isolated from olive flounder (Paralichthys olivaceus). FEMS Microbiol Lett 2009, 293(1):20-27.

8. Yuasa K, Kitancharoen N, Kataoka Y, Al-Murbaty FA: Streptococcus iniae, the causative agent of mass mortality in rabbitfish Siganus canaliculatus in Bahrain. J Aquat Anim Health 1999, 11:87-93.

9. Eldar A, Ghittino C: Lactococcus garvieae and Streptococcus iniae infections in rainbow trout Oncorhynchus mykiss: similar, but different diseases. Dis Aquat Organ 1999, 36(3):227-231.

10. Lahav D, Eyngor M, Hurvitz A, Ghittino C, Lublin A, Eldar A: Streptococcus iniae type II infections in rainbow trout Oncorhynchus mykiss. Dis Aquat Org 2004, 62:177-180.

11. Eldar A, Bejerano Y, Livoff A, Horovitcz A, Bercovier H: Experimental streptococcal meningo-encephalitis in cultured fish. Vet Microbiol 1995, 43(1):33-40.

12. Weinstein MR, Litt M, Kertesz DA, Wyper P, Rose D, Coulter M, McGeer A, Facklam R, Ostach C, Willey BM, Borczyk A, Low DE: Invasive infections due to a fish pathogen, Streptococcus iniae. S. iniae Study Group. N Engl J Med 1997, 337:589-594.

13. Wooldridge KG, Williams PH: Iron uptake mechanisms of pathogenic bacteria. FEMS Microbiol Rev 1993, 12(4):325-348.

14. Litwin CM, Calderwood SB: Role of iron in regulation of virulence genes. Clin Microbiol Rev 1993, 6(2):137-149.

15. Noya F, Arias A, Fabiano E: Heme compounds as iron sources for nonpathogenic rhizobium bacteria. J Bacterio/ 1997, 179(9):3076-3078.

16. Tam R, Saier MHJ: Structural, functional, and evolutionary relationships among extracellular solute binding receptors of bacteria. Microbiol Mol Biol Rev 1993, 57(2):320-346.

17. Holland IB, Blight MA: ABC-ATPases, adaptable energy generators fuelling transmembrane movement of a variety of molecules in organisms from bacteria to humans. J Mol Biol 1999, 293(2):381-399.

18. Saurin W, Hofnung M, Dassa E: Getting in or out: early segregation between importers and exporters in the evolution of ATP binding cassette (ABC) transporters. J Mol Evol 1999, 48(1):22-41.

19. Schultz J, Milpetz F, Bork P, Ponting CP: SMART, a simple modular architecture research tool: identification of signaling domains. Proc Natl Acad Sci 1998, 95(11):5857-5864.

20. Letunic I, Doerks T, Bork P: SMART 6: Recent updates and new developments. Nucleic Acids Res 2009, 37:229-320.

21. Walker JE, Saraste M, Runswick MJ, Gay NJ: Distantly related sequences in the $\alpha$ - and $\beta$-subunits of ATP synthase, myosin, kinases and other ATP requiring enzymes and a common nucleotide bingding fold. EMBO J 1982, 1:945-951.

22. Saraste $M$, Sibbald PR, Wittinghofer A: The P-loop, a common motif in ATP- and GTP- binding proteins. Trends Biochem Sci 1990, 15(11):430-434

23. Hyde SC, Emsley P, Hartshorn MJ, Mimmack MM, Gileadi U, Pearce SR, Gallagher MP, Gill DR, Hubbard RE, Higgins CF: Structural model of ATPbinding proteins associated with cystic fibrosis, multidrug resistance and bacterial transport. Nature 1990, 346:362-365.

24. Ames FLG, Mimira CS, Shyamala V: Bacterial periplasmic permeases belong to a familu of transport proteins operating from Escherichia coli to human: traffic ATPase. FEMS Microbiol Rev 1990, 75(4):429-446.

25. Linton KJ, Higgins CF: The Escherichia coli ATP-binding cassette (ABC) proteins. Mol Microbiol 1998, 28(1):5-13.

26. Hutchings MI, Palmer T, Harrington DJ, Sutcliffe LC: Lipoprotein biogenesis in Grampositive bacteria: knowing when to hold 'em, knowing when to fold 'em. Trends Microbiol 2008, 17(1):13-21.

27. Sutcliff I, Russell RR: Lipoproteins of Gram-positive bacteria. J Bacteriol 1995, 177(5):1123-1128.

28. Fuhrhop JH, Smith KM: Porphyrins and metalloporphyrins. In Laboratory methods. Edited by: Smith KM. New York: Elsevier Press; 1975:804-807.

29. Thammavongsa V, Kern JW, Missiakas DM, Schneewind O: Staphylococcus aureus synthesizes adenosine to escape host immune responses. J Exp Med 2009, 206(11):2417-2427. 
30. Otto BR, Sparrius M, Verweij-van VAM, MacLaren DM: Iron-regulated outer membrane protein of Bacteroides fragilis involved in heme uptake. Infect Immun 1990, 58(12):3954-3958.

31. Litwin CM, Calderwood SB: Role of iron in regulation of virulence genes. Clin Microbiol Rev 1993, 6(2):137-149.

32. Brooks HJL, O'Grady F, Mcsherry MA, Cattel WR: Uropathogenic properties of Escherichia coli in recurrent urinary-tract infection. J Med Microbiol 1980, 13:57-68.

33. Crosa $\mathrm{JH}$ : A Plasmid associated with virulence in the marine fish pathogen Vibro anguillarum specifies an iron-sequestering system. Nature 1980, 284:566-568.

34. DeBoy JM, Wachsmuth IK, Davis BR: Hemolytic activity in enterotoxigenic and nonenterotoxigenic strains of Escherichia coli. J Clin Microbiol 1980, 12(2):193-198.

35. Margaret A, Linggood, Ingram PL: The role of alpha haemolysin in the virulence of Escherichia coli for mice. J Med Microbiol 1982, 15(1):23-30.

36. Waalwijk C, MacLaren DM, de Graaff : In vivo function of hemolysin in the nephropathogenicity of Escherichia coli. Infec Immun 1983, 42(1):245-249.

37. Williams PH: Novel iron uptake system specified by ColV plasmids: an important component in the virulence of invisive strains of Escherichia coli. Infec Immun 1979, 26:925-932.

38. Crosa JH, Walsh CT: Genetics and Assembly Line Enzymology of Siderophore Biosynthesis in Bacteria. Microbiol Mol Biol R 2002, 66(2):223-249.

39. Sun XS, Ge RG, Chiu JF, Sun HZ, He QY: Lipoprotein MtsA of MtsABC in Streptococcus pyogenes primarily binds ferrous ion with bicarbonate as a synergistic anion. FEBS Microbiol Lett 2008, 582(9):1351-1354.

40. Desvaux M, Dumas E, Chafsey I, Hébraud M: Protein cell surface display in Gram-positive bacteria: from single protein to macromolecular protein structure. FEMS Microbiol Lett 2006, 256(1):1-15.

41. Holland IB, Cole SPC, Kuchler K, Higgins CF: ABC proteins: from bactria to man London. Academic 2003, 279-293.

42. Davidson AL, Chen J: ATP-binding cassette transporters in bacteria. Annu Rev Biochem 2004, 73:241-268.

43. Hollenstein K, Dawson RJP, Locher KP: Structure and mechanism of ABC transporter proteins. Curr Opin Struc Biol 2007, 17(4):412-418.

44. Braun V, Wu HC: Lipoproteins, structure, function, biosynthesis and model for protein export. New Compr Biochem 1994, 27:319-341.

45. Zhou SM, Xie MQ, Zhu XQ, Ma Y, Tan ZL, Li AX: Identification and genetic characterization of Streptococcus iniae strains isolated from diseased fish in China. J Fish Dis 2008, 31(11):869-875.

46. Tai GH, Gao y, Shi M, Zhang XY, He SP, Chen ZL, An CC: SiteFinding-PCR: a simple and efficient PCR method for chromosome walking. Nucleic Acids Research 2005, 33(13):e122.

47. Regulations for the administration of affairs concerning experimental animals: the State Council of the People's Republic of China and the State Science and Technology Commission. Peking; 1988.

48. Bray BA, Sutcliffe IC, Harrington DJ: Expression of the MtsA lipoprotein of Streptococcus agalactiae A909 is regulated by manganese and iron. Antonie Van Leeuwenhoek 2009, 95:101-109.

49. Cockayne A, Hill PJ, Powell NBL, Bishop K, Sims C, Williams P: Molecular cloning of a 32-kilodalton lipoprotein component of a novel ironregulated Staphylococcus epidermidis ABC transporter. Infect Immun 1998, 66(8):3767-3774.

50. Laemmli UK: Cleavage of structural proteins during the assembly of the head of bacteriophage T4. Nature 1970, 227:680-685.

51. Tai SS, Yu C, Lee JK: A solute binding protein of Streptococcus pneumoniae iron transport. FEMS Microbiol Lett 2003, 220(2):303-308.

52. Bolotin S, Fuller JD, Bast DJ, Azavedo JCSD: The two-component system sivS/R regulates virulence in Streptococcus iniae. FEMS Immunol Med Microbiol 2007, 51(3):547-554.

53. Homonylo-McGavin MK, Lee SF: Role of the terminus in antigen P1 surface localization in Streptococcus mutans and two related cocci. J Bacteriol 1996, 178(3):801-807.

54. Lei BF, Wei CJ, Tu SC: Action mechanism of antitubercular isoniazid: activation by Mycobacterium tuberculosis KatG, isolation, and characterization of InhA inhibitor. J Biol Chem 2000, 275:2520-2526.
55. Lei BF, Smoot LM, Menning HM, Voyich JM, Kala SV, Deleo FR, Reid SD, Musser JM: Identification and Characterization of a Novel HemeAssociated Cell Surface Protein Made by Streptococcus pyogenes. Infect Immun 2002, 70(8):4494-4500.

doi:10.1186/1471-2180-10-309

Cite this article as: Zou et al:: A solute-binding protein for iron

transport in Streptococcus iniae. BMC Microbiology 2010 10:309.

\section{Submit your next manuscript to BioMed Central and take full advantage of:}

- Convenient online submission

- Thorough peer review

- No space constraints or color figure charges

- Immediate publication on acceptance

- Inclusion in PubMed, CAS, Scopus and Google Scholar

- Research which is freely available for redistribution 